\title{
Lumen
}

Selected Proceedings from the Canadian Society for Eighteenth-Century Studies

\section{Topoï des identités nationales dans la nouvelle française du XVIII $^{\mathrm{e}}$ siècle}

\section{Monique Moser-Verrey}

Volume 21, 2002

URI : https://id.erudit.org/iderudit/1012275ar

DOI : https://doi.org/10.7202/1012275ar

Aller au sommaire du numéro

Éditeur(s)

Canadian Society for Eighteenth-Century Studies / Société canadienne d'étude du dix-huitième siècle

ISSN

1209-3696 (imprimé)

1927-8284 (numérique)

Découvrir la revue

Citer cet article

Moser-Verrey, M. (2002). Topoï des identités nationales dans la nouvelle française du XVIII siècle. Lumen, 21, 199-211.

https://doi.org/10.7202/1012275ar

Copyright (C Canadian Society for Eighteenth-Century Studies / Sociéte canadienne d'étude du dix-huitième siècle, 2002
Ce document est protégé par la loi sur le droit d'auteur. L'utilisation des services d'Érudit (y compris la reproduction) est assujettie à sa politique d'utilisation que vous pouvez consulter en ligne.

https://apropos.erudit.org/fr/usagers/politique-dutilisation/ 


\section{Topoï des identités nationales dans la nouvelle française du XVIII ${ }^{\mathrm{e}}$ siècle}

Dans le cadre d'une réflexion sur la mémoire et sur l'identité, il m'importe de scruter ici un élément récurrent de la caractérisation du personnage de fiction, soit l'identité nationale. Ma recherche porte, en effet, sur une systématisation des marques textuelles qui soutiennent la visualisation du corps romanesque. Pour ce faire, je distingue les marques d'identité de celles qui illustrent la sensibilité et la sociabilité du personnage. Or les identités nationales, souvent évoquées, me semblent relever du topos, dans la mesure où elles convoquent une série d'idées préconçues que le récit peut actualiser sur différents plans pour conforter ou surprendre les attentes du lecteur. Qu'en est-il de ces attentes au XVIII ${ }^{e}$ siècle et comment la nouvelle française y répond-elle?

L'Encyclopédie s'intéresse aux identités nationales. Dans l'article «Nation» on trouve, par exemple, la remarque suivante, accompagnée d'un renvoi à l'article «Caractère»:

Chaque nation a son caractere particulier: c'est une espece de proverbe que de dire, leger comme un françois, jaloux comme un italien, grave comme un espagnol, méchant comme un anglois, fier comme un écossois, ivrogne comme un allemand, paresseux comme un irlandois, fourbe comme un grec, \&c. Voyez CARACTÈRE (Encycl., XI, 1765, p. 36, b) ${ }^{1}$.

Dans sa discussion du «CARACTÈRE, en Morale», parue dans le tome II en 1751, D'Alembert s'arrêtait uniquement au caractère des Français et

1 L'orthographe de toutes les citations de l'Encyclopédie correspond à celle de la version reproduite dans Diderot et D'Alembert (édit.), Encyclopédie ou Dictionnaire raisonné des sciences, des arts et des métiers. Compact Edition, Vol. I-V, New York et Paris, Pergamon Press, 1969. Les références seront incluses dans le corps du texte. 
n'évoquait les Athéniens et les Germains que pour affirmer l'existence d'un "fond de caractere» inchangé depuis les remarques de Démosthène et de Tacite à l'endroit de ces nations. Son propos a une portée politique comme on le voit immédiatement par les derniers traits évoqués: «le caractere des François est la légereté, la gaieté, la sociabilité, l'amour de leurs rois \& de la monarchie même, \&c.» (Encycl., II, 1751, p. 666, a). Il s'agit bien, par la suite, de faire la guerre au despotisme et de faire l'éloge de «la Philosophie», à laquelle on doit rien de moins que le salut des États, comme le montre «le célebre M. de Voltaire, dans son admirable Essai sur le siecle de Loüis XIV» (Encycl., II, 1751, p. 666, b). En l'occurrence, l'esprit éclairé est censé avoir persuadé les religieux «qu'ils sont sujets du Roi avant que d'être serviteurs du Pape» (Encycl., II, 1751, p. 666, b). Cette adhésion à la nation est signalée comme un grand changement dans les moars des Français. On verra que, tout au long de la seconde moitié du siècle, la fierté nationale des Français se précise et ne reste pas sans effet sur la façon d'apprécier les étrangers.

Le corpus de nouvelles que j'ai interrogé pour analyser la caractérisation des personnages étrangers est simplement la collection des Nouvelles françaises $d u X V I I I^{\mathrm{e}}$ siècle présentée par Jacqueline Hellegouarc'h et disponible en livre de poche ${ }^{2}$. Un bref coup d'œil jeté aux titres de la cinquantaine de textes réunis dans cette collection révèle que plusieurs nouvelles sont identifiées par la mention de l'identité nationale qu'elles mettent en jeu. C'est vrai au début du siècle dans les Illustres Françaises de Robert Challe ou dans Cosi-Sancta, un petit mal pour un grand bien. Nouvelle africaine de Voltaire. Mais cela devient plus insistant à partir des années soixante, quel'on pense à Saint-Lambert, Deux amis, conte iroquois, à Cazotte, Rachel, ou la Belle Juive, nouvelle héroïque espagnole, à Florian, Claudine, nouvelle savoyarde et Valérie, nouvelle italienne, ou à Mercier de Compiègne, la Sorcière de Verberie; nouvelle française. Ce qui frappe, bien sûr, c'est la mention explicite du genre qui semble lier l'identité nationale à une question de poétique.

La suite de mon propos servira à démontrer que l'identité nationale des personnages romanesques peuplant la nouvelle française ne relève pas tant d'une observation de première main des nations concernées que du topos, soit emprunté à la banque de proverbes déjà citée, soit construit à partir de la poésie et de la littérature des autres nations elles-mêmes,

2 Jacqueline Hellegouarc'h (édit.), Nouvelles françaises $d u$ XVIII siècle, vol. I: De Voltaire à Voisenon et vol. II: De Marmontel à Potocki, Paris, Librairie générale française, coll. «Le livre de poche», 705 et 709, 1994. Toutes les références seront incluses dans le corps du texte. 
selon l'appréciation d'un poéticien français les ayant dépeintes. L'émergence d'un intérêt plus marqué pour les nationalités à partir des années soixante-dix coïncide, en effet, avec une reformulation de l'idée de poésie que l'on doit à Jean-François Marmontel, reformulation qui tient compte, au-delà de la poésie des Anciens, de la poésie des nations de l'Europe moderne.

L'article «Poésie», qui servira de point de référence à ma réflexion sur la construction de l'identité étrangère dans l'écriture de la nouvelle française, ne remonte pas à la Poétique française de Marmontel, encore que la perspective européenne soit déjà sensible dans ce premier ouvrage paru en $1763^{3}$. Cela est par contre le cas de l'article «Poétique», comme le remarque Annie Becq dans un article récent où elle appelle de ses vœux «L'étude précise des modalités de dépeçage de ce texte ${ }^{4} . »$ Pour sa part, Michael Cardy s'intéresse aussi à la première parution des articles que l'on retrouvera ensuite dans les Éléments de littérature de Marmontel, mais il se trompe, lorsqu'il classe l'article «Poésie» parmi ceux censés être "completely new ${ }^{5}$ » en 1786 dans le troisième volume de Grammaire et littérature inclus dans l'Encyclopédie méthodique. En fait, cet article avait déjà paru en 1777 dans le $I^{\mathrm{e}}$ tome du Supplément à l'Encyclopédie avant d'être repris dans l'Encyclopédie méthodique et dans les Éléments de littérature. A cette époque, Herder venait d'être appelé à Weimar, ayant produit au début des années soixante-dix sa théorie du langage et de la poésie compris comme expressions d'une identité nationale ${ }^{6}$.

L'article «Poésie» de Marmontel témoigne d'une vision vitaliste ou "primitiviste ${ }^{7}$ » de la poésie qui est absolument étrangère au premier article «Poésie» paru en 1765 dans le XII tome de l'Encyclopédie et signé

3 Voir l'Avant-propos où il est question de «la renaissance des Lettres en Europe», dans Jean-François Marmontel, Poétique françoise, Lesclapart, Paris, 1763, p. 1.

4 Annie Becq, «La Poétique Française de Jean-François Marmontel», dans Kees Meerhoff et Annie Jourdan (édit.), Mémorable Marmontel, 1799-1999, Amsterdam et Atlanta, GA, Rodopi, coll. «CRIN», 35, 1999, p. 162.

5 Michael Cardy, The Literary Doctrines of Jean-François Marmontel, Studies on Voltaire and the Eighteenth Century, CCX, 1982, p. 16.

6 Voir Abhandlung über den Ursprung der Sprachen, 1772 (Essai sur l'origine des langues) et le collectif Von deutscher Art und Kunst, 1773 (De l'Allemagne et de son art). Comme me le signalait Jacques Wagner, à qui je suis redevable de cette remarque, la pensée de Herder commençait alors à exercer de la pression sur les esprits.

7 Roland Mortier, l'Originalité. Une nouvelle catégorie esthétique au Siècle des lumières, Genève, Droz, 1982, p. 173. 
par le chevalier de Jaucourt. Tandis que Jaucourt tente, à la suite de Batteux, de définir l'essence de la poésie, Marmontel se propose d'en écrire les révolutions. Il veut

considérer la poésie comme une plante; examiner pourquoi, indigene dans certains climats, on l'y a vu naître \& fleurir d'elle-même; pourquoi, étrangere par-tout ailleurs, elle n'a prospéré qu'à force de culture; ou pourquoi, sauvage \& rébelle, elle s'est refusée aux soins qu'on a pris de la cultiver; enfin pourquoi, dans le même climat, tantôt elle a été florissante \& féconde, tantôt elle a dégénéré (Suppl., IV, 1777, p. 426, a et b) ${ }^{8}$.

La référence aux climat, comme facteur déterminant pour l'épanouissement de la poésie, rejoint entièrement le lieu commun qui sert d'explication à la variété des caractères nationaux. D'Alembert en fait mention dans l'article déjà cité, avant d'élaborer sur l'influence des formes de gouvernement: "Il y a grande apparence que le climat influe beaucoup sur le caractere général» (Encycl., II, 1751, p. 666, a). Qu'en est-il enfin des identités nationales, si l'on en croit non pas tant de rapides proverbes, mais l'étude des révolutions de la poésie parmi les nations?

Selon la thèse de Marmontel, la poésie se développe là où les conditions lui sont favorables. Ce fut le cas dans la Grèce ancienne, car grâce au climat le naturel du peuple y était vif et sensible, sa langue naturellement poétique, «figurée, mélodieuse, riche, abondante, variée, \& habile à tout exprimer» (Suppl., IV, p. 427, a). Enfin, la poésie des Grecs reçut de la musique «ses premieres loix, la mesure et le mouvement» (Suppl., IV, p. 427, b). L'éloge de la poésie grecque s'étend sur plus de trois pages du Supplément à l'Encyclopédie pour se conclure par l'affirmation qu'en Grèce «la nature, la fortune, l'opinion, les loix, les mœurs, tout s'étoit accordé pour la favoriser» (Suppl., IV, p. 430, a). Et Marmontel de poursuivre: «Il sera bien aisé de voir à présent dans quel autre pays du monde elle a trouvé plus ou moins de ces avantages» (Suppl., IV, p. 430, a). Il est intéressant de remarquer que Marmontel utilise ici le singulier «quel autre pays» et l'on verra plus loin où il veut en venir.

Les nations passées au crible de la combinatoire des circonstances favorables à la poésie comprenant la nature, la fortune, l'opinion, les lois et les mœars sont: Rome, puis l'Italie moderne, l'Espagne, l'Angleterre,

8 Voir Jean-François Marmontel, «Poésie», Élémens de littérature, dans CEuvres complètes, tome V, $1^{\text {re }}$ partie, Genève, Slatkine Reprints, 1968, p. 1-39. Nous citons cet article selon le Supplément à l'Encyclopédie ou Dictionnaire raisonné des sciences, des arts et des métiers, Amsterdam, M. M. Rey, 1777, tome VI, p. 426-440. 
l'Allemagne et pour conclure la France. J'ai eu l'occasion ailleurs ${ }^{9}$ de m'interroger sur la transformation du regard jeté sur le corps romanesque des Français ou plus exactement des Françaises, car elles sont beaucoup plus souvent l'objet de la focalisation dans la nouvelle que leurs partenaires mâles. A vrai dire, elles sont bien loin d'être toutes légères et gaies. En fait, elles le sont uniquement dans la nouvelle libertine. Mais cette observation ne change rien au topos qui est tenace. Voici ce qu'en dit Marmontel dans ce qu'il appelle son «histoire naturelle de la poésie» (Suppl., IV, p. 440):

La premiere cause de la faveur \& des succès qu'eut la poésie dans un climat qui n'étoit pas le sien, fut le caractere d'un peuple curieux, léger \& sensible, passionné pour l'amusement, \&, après les Grecs, le plus susceptible qui fût jamais d'agréables illusions. Mais ce n'eût été rien, sans l'avantage prodigieux pour les muses de trouver une ville opulente \& peuplée, qui fût le centre des richesses, du luxe \& de l'oisiveté, le rendez-vous de la partie la plus brillante de la nation, attirée par l'espérance de la faveur \& de la fortune, \& par l'attrait des jouissances. Il est plus que vraisemblable, que s'il n'y avoit pas eu un Paris, la nature auroit inutilement produit un Corneille, un Racine, \&c. (Suppl., IV, p. 438-439)

En effet, dans la suite de l'article, Marmontel traite avant tout de Molière qui incarne au plus près l'avantageux caractère national des Français. Ce sont vraiment les sommets de la littérature nationale qui, selon cette vision, articulent au mieux le caractère national. Ici la France se confond avec Paris, qui «devoit être naturellement le grand théâtre de la comédie moderne» (Suppl., IV , p. 439, a), pleine de légèreté et de gaieté, à l'image des Français et vice versa. Dans le corpus de nouvelles étudié, on trouve certes des auteurs qui confirment le topos de la légèreté et de la gaieté françaises, que l'on pense à Marivaux, Crébillon, Vivant Denon et Rétif de la Bretonne. Mais les Français et les Françaises sérieux et sombres n'y manquent pas, que l'on pense au Spleen de Besenval, à l'Histoire $d u$ marquis de Cressy de Mme Riccoboni, aux Deux amis de Bourbonne de Diderot, et j'en passe.

La lecture croisée de la caractérisation des poésies nationales proposée par Marmontel et de la caractérisation des personnages étrangers dans les Nouvelles françaises est nettement plus concluante. Ma démonstration suivra maintenant les identités étrangères selon l'ordre dans

9 «Le langage du corps romanesque des Illustres Françaises (1713) à la Sorcière de Verberie (1798)», Eighteenth Century Fiction, 13, 2-3, janvier-avril 2001, p. 349-388. 
lequel Marmontel les présente dans son article. Cet ordre est déterminé par l'apparition de grands auteurs au sein des nations considérées. L'Italie moderne semble donc avoir réuni d'excellentes conditions pour voir s'épanouir la poésie épique au XVI ${ }^{\mathrm{e}}$ siècle. Sa langue est notamment riche et mélodieuse:

Aux avantages de cette langue, déja cultivée par Dante, Boccace et Pétrarque, se joignoient, en faveur de la poésie épique, l'esprit de superstition dont l'Italie étoit le centre, les mœurs de la chevalerie qui avoit été l'héroïsme Gaulois, \& qui restoit encore à peindre; \& l'intérêt vif \& récent de l'expédition des croisades, sujet héroïque \& sacré, \& d'un intérêt à-la-fois religieux \& profane, sujet par-là peut-être unique dans toute l'histoire moderne.

L'Arioste, dans un poëme héroï-comique, le Tasse, dans un poëme sérieux \& vraiment épique, profiterent de ces avantages, tous deux en hommes de génie (Suppl., IV, p. 432, b).

Ce qui intéresse en Italie par rapport à la poésie, c'est le merveilleux, tant celui de la magie que celui de la religion. On est renvoyé ici à l'article "Merveilleux», où l'Arioste est cité pour illustrer le merveilleux naturel et le Tasse pour illustrer le merveilleux surnaturel. Selon Marmontel, il est «raisonnable et permis» d'utiliser le merveilleux dans certaines conditions.

La seule attention qu'on doit avoir est de saisir bien au juste l'opinion des peuples à la place desquels on veut nous mettre, afin de ne pas faire du merveilleux un usage dont eux-mêmes ils seroient blessés (Suppl., III, p. 907, a).

Le corpus de nouvelles étudié ne met l'Italie à l'honneur que dans deux textes tirés des Nouvelles nouvelles (1792) de Florian. Avant cela, on trouve des échos de la langue italienne dans Cosi-Sancta (1715) de Voltaire, où le mari, Capito, incarne le préjugé proverbial de l'Italien jaloux. Bien que la scène soit à Hippone, en Afrique du Nord, ce mari est présenté dès la première page de la nouvelle comme «un petit homme bourru et chagrin [...], jaloux d'ailleurs comme un Vénitien» (I, 80). L'Histoire intéressante (1740) de l'abbé Prévost, parue dans le Pour et le contre, a aussi pour héros un mystérieux Italien, mais tout se passe à Amsterdam, où personne ne parvient à percer son secret. Venu de Rome, il retournera à Rome après avoir perdu sa compagne et dignement fait élever son enfant. Le seul élément qui le distingue comme Italien est son langage, comme en témoigne le portrait qu'en brosse le narrateur:

un homme de quarante ans, d'une taille haute et épaisse, la physionomie commune, mais avec un air de bonté néanmoins qui intéressait tout d'un coup 
en sa faveur; parlant mal notre langue, et né comme je le remarquai aussitôt à son langage, dans quelque partie de l'Italie. Les manières d'ailleurs assez douces et assez polies pour faire juger que, de quelque condition qu'il fût, il avait reçu une fort bonne éducation (I, p. 215-216).

Les bruits qui courent à son endroit, «maître d'hôtel italien» ou "prélat romain» (I, p. 217), ne se confirment pas. Son désespoir à la suite du suicide de la baronne, sa femme ou sa compagne, ne le rapporte pas à un quelconque caractère national, mais témoigne d'un grand amour meurtri qu'il cachera pendant sept ans «dans un couvent de Westphalie» (I, p. 222) avant de retourner dans sa patrie. L'itinéraire du personnage, ses mœurs, son éducation et le mystère dont il s'entoure font davantage penser à un religieux, serviteur du pape, qu'à un baron identifié à la nation italienne dont il pourrait difficilement être question à cette époque.

Tout se passe, par contre, dans Valérie, nouvelle italienne, comme si le narrateur suivait à la lettre les idées de Marmontel sur les particularités nationales et l'usage du merveilleux en poésie. Il prend soin de situer son histoire de revenants dans un contexte qui rend le merveilleux acceptable. D'abord, il affronte les "esprits forts» en citant Plutarque, Pline et Agrippa d'Aubigné, qui auraient vu des morts revenir les hanter. Puis, il situe la veillée dans un château du Languedoc où il se trouve avec de jeunes Languedociennes qui, dit-il, «ne manquaient pas d'imagination, chose assez commune dans notre pays, [et] se plaisaient beaucoup aux histoires de revenants» (II, p. 690):

Nous avions avec nous une jeune Italienne nommée Valérie d'Orsini, que sa mauvaise santé avait fait venir à Montpellier pour consulter nos médecins. [...] Cette jeune étrangère était fort aimable. [...] Ses grands yeux noirs étaient languissants; son regard inspirait la tendresse; et sa beauté, sa grâce touchante semblaient acquérir un charme de plus de la pâleur éternelle qui couvrait toujours son visage. Ses lèvres mêmes n'étaient pas exemptes de cette pâleur: lorsque Valérie parlait, on croyait voir s'animer une statue d'albâtre; lorsqu'elle ne parlait pas, elle n'attirait pas moins les regards, et l'on trouvait alors vraisemblable l'aventure de Pygmalion (II, p. 691).

On s'en doute, l'Italienne est un revenant. Elle a vécu à Florence et y est morte dans des circonstances révoltantes auxquelles l'amour du comte d'Orsini et sa profonde foi religieuse l'ont heureusement arrachée. Revenue à la vie grâce au «miracle de l'amour» (II, p. 703), elle donne à l'Église, durant la semaine sainte, un spectacle qui permet d'apprécier la superstition du peuple italien et qui est capable de soutenir un intérêt à la fois religieux et profane où l'héroïsme et le sacré se rencontrent, tout 
comme l'Arioste et le Tasse ont su le faire selon Marmontel. On n'en est plus au temps des croisades et pourtant Valérie est en croisade pour libérer les droits de l'amour contre ceux d'un pouvoir marital usurpé. Voici la scène qui se produit au moment où ses parents et son mari, Héraldi, qui se recueillent dans la chapelle où elle fut enterrée, l'aperçoivent:

je veux fuir, la foule m'arrête. Héraldi s'approche de moi; il étend déjà la main pour me saisir par mes habits. J'étais perdue, si, dans le moment, l'amour ne m'avait inspirée. - Arrête, lui dis-je d'une voix que je m'efforçai de rendre terrible; respecte, du moins après son trépas, celle que tu trompas pendant sa vie. Toi seul as causé ma mort. Laisse-moi, pleure ton crime, et fléchis le courroux du ciel.

Après avoir dit ces mots, qu'Héraldi, glacé de terreur, écouta sans oser faire un mouvement, j'enveloppai ma tête dans mon voile, et je marchai d'un pas tranquille vers la porte de l'église: le peuple s'ouvrait devant moi (II, 706-707).

Revenant, libérée des injustices de l'Ancien Régime, Valérie est surtout une Italienne digne de l'art des plus grands poètes de sa nation.

Les Espagnols sont beaucoup plus présents dans le corpus de nouvelles étudié que ne le sont les Italiens. Dans l'Histoire de Monsieur de Jussy et de Mademoiselle Fenouil de Challe, nouvelle qui ouvre le premier recueil, on trouve déjà un personnage épisodique qui illustre la barbarie de cette nation. Selon Marmontel, qui a en vue la littérature du XVII siècle espagnol en ciblant avant tout Lope de Vega et Calderon: «Le goût des Espagnols pour le spectacle donna plus d'émulation à la poésie dramatique; \& la tragédie pouvoit encore trouver des sujets dignes d'elle dans l'histoire de leur pays» (Suppl., IV, p. 434, a). Si dans la tragédie espagnole on trouve des peintures fortes et des scènes terribles, c'est à cause du «faux goût des Orientaux \& des Barbares» qui se sont longtemps disputé le territoire de l'Espagne. Aussi «l'amour [est] luimême en Espagne plus fier, plus fougueux, plus jaloux, plus sombre dans sa jalousie, et plus cruel dans ses vengeances que dans aucun autre pays du monde» (Suppl., IV, p. 434, a). Tout cela est connu bien avant que Marmontel ne le dise, puisqu'il s'appuie pour développer son argumentation sur une longue citation de Lope de Vega lui-même. Challe pouvait donc bien confier à un rival jaloux le soin de tuer à Madrid un certain $M$. de Jussy, trépas dont le héros du même nom profita pour se faire passer pour mort. Comme ce héros est aussi le narrateur de ses aventures, il ne manque pas de souligner qu'il avait averti son compatriote libertin du danger qu'il courait en se montrant léger «dans un pays où la jalousie règne, et où les maris se croient tout permis pour venger l'honneur qu'ils croient qu'on leur ôte, par le 
commerce qu'on peut avoir avec leurs femmes, ou avec une autre de leur famille» (I, p. 51).

La violence et l'audace caractérisent décidément l'Espagnol. On en trouve un autre exemple dès les premières pages des deux premières "Journées» du Manuscrit trouvéa Saragosse de Jean Potocki, fragment par lequel se clôt notre collection de nouvelles françaises du XVIII ${ }^{\mathrm{e}}$ siècle. Est-il bien français ou plutôt européen, ce texte d'un auteur polonais qui imite à la perfection la verve du roman picaresque espagnol? Quoi qu'il en soit, on est près d'une auberge aux abords de la redoutable Sierra Morena qu'affrontait jadis Don Quichotte. Les mises en garde de l'aubergiste ne retiennent pas le jeune capitaine aux Gardes wallonnes du roi don Felipe Quinto (1683-1746), qui lui répond: «les lois sacrées de l'honneur me prescrivaient de me rendre à Madrid par le chemin le plus court, sans demander s'il était le plus dangereux» (II, p. 748). Puis, ce narrateur intrépide poursuit son récit en ajoutant: «Il en eût dit davantage, mais je piquai des deux et ne $\mathrm{m}$ 'arrêtai que lorsque je me crus hors de la portée de ses remontrances» (II, p. 748). On est en pleine parodie, mais le topos de l'honneur lié à l'identité espagnole masculine n'en est pas moins actualisé.

L'image des femmes espagnoles n'est, par contre, pas vraiment avantageuse dans les nouvelles étudiées. "Sèche et encore plus basanée» (I, p. 339), Mariquilla, Espagnole qui appartient à la maison d'Omar dans le Cousin de Mahomet ou la folie salutaire (1742) de Fromaget, se ruine en avances très intelligibles pour un héros qui feint de n'y rien comprendre. Plus tard, dans la nouvelle Joli-piéd (1780) de Rétif de la Bretonne, la sécheresse mentionnée par Fromaget semble correspondre au goût espagnol en général, car "partout hors en Espagne une belle gorge à son prix» (II, p. 465). On est enfin à la veille de la Révolution, lorsque Cazotte emprunte à l'Espagne la trame de sa nouvelle historique intitulée Rachel, ou la belle Juive (1788). Dans cette nouvelle dont le sujet avait déjà été traité à de nombreuses reprises en Espagne, entre autres par Lope de Vega lui-même ${ }^{10}$, le "faux goût barbare», dont Marmontel accuse les Espagnols, culmine dans le meurtre de la belle Juive qui avait détourné le roi Alphonse de son devoir. En l'occurrence, le caractère grave et incorruptible du peuple espagnol a raison des machinations cabalistiques du rabbin Ruben et du pouvoir de séduction de la belle Rachel pour ramener le roi dans le droit chemin.

10 Jacqueline Hellegouarc' $h$ retrace la fortune littéraire de la Juive de Toledo, amante du roi de Castille Alphonse VIII (1155-1214) dans la note 2 (II, p. 612-613). 
On peut s'étonner de la dévalorisation de l'identité espagnole tant dans les nouvelles que dans la perspective de Marmontel, dont le recours est assez pervers, puisqu'il cite longuement Lope de Vega pour dénigrer l'art du grand dramaturge. Dans ce passage, rendu en vers français, celui-ci dénonce lui-même la barbarie de son public qui conditionne son écriture:

Je me vois obligé de servir l'ignorance,

D'enfermer sous quatre verroux

Sophocle, Euripide \& Térence.

J'écris en insensé, mais j'écris pour des foux (Suppl., IV, p. 434, b).

Tout se passe comme si, la gravité espagnole étant aux antipodes de la légèreté française, il fallait vigoureusement rejeter ce type d'altérité. Mais, si on lit bien attentivement la suite de l'article de Marmontel, on voit où le bât blesse:

La premiere piece vraiment comique qui parut sur le théâtre français, depuis l'Avocat Patelin, ce fut le Menteur de Corneille, piece imitée de l'Espagnol, de Lopes de Vega ou de Roxas: M. de Voltaire le met en doute; \& il observe, à propos du Menteur, que le premier modele du vrai comique, ainsi que du vrai tragique (le Cid), nous est venu des Espagnols, \& que l'un \& l'autre nous a été donné par Corneille (Suppl., IV, p. 439, b).

La vigueur du modèle espagnol menace légèrement la vanité française par la richesse des sources auxquelles Cazotte et Potocki puisent encore hardiment. Il importe donc au poéticien de souligner en quoi les grands classiques français de la seconde moitié du XVII ${ }^{\mathrm{e}}$ siècle se distancient de leurs éminents prédécesseurs espagnols du début de ce même siècle. En définitive, Marmontel trouve le génie français "peu inventif», mais il reconnait qu'il «s'applique sans relâche à tout perfectionner» et qu'il «a été la cause constante des progrès de la poésie dans un climat qui ne sembloit pas fait pour elle» (Suppl., IV, p. 440, a).

Il apparaît que si la poésie française l'emporte sur les autres poésies nationales en atteignant un «si haut point de splendeur» (Suppl., IV, p. 440 , a), elle le doit au raffinement du goût que les circonstances de son épanouissement ont rendu possible. Dès lors, elle peut inspirer aux nations voisines une sensibilité pour «les véritables beautés de l'art». Et Marmontel d'affirmer: «Ce goût de la belle nature, les Anglois le prirent en France à la cour de Louis de Grand, \& le porterent dans leur patrie. Ce fut à Moliere, à Racine, à Despréaux, qu'ils durent Dryden, Pope, Adisson [sic]» (Suppl., IV, p. 435, a). A vrai dire, Marmontel s'étonne que le peuple anglais, sérieux, peu sensible, mélancolique et très occupé de 
ses intérêts politiques ait produit "d'excellens poëtes", mais il reconnaît que la poésie conforme au génie national des Anglais, «toute inculte \& sauvage qu'elle est, peut avoir encore sa beauté» (Suppl., IV, p. 435, a), par la force, la hardiesse, l'énergie, la franchise, la vérité naïve et autres vertus qui compensent le manque de grâce, d'élégance et de régularité.

Par ailleurs, Marmontel fait dépendre du goût du peuple censé dominer en Angleterre et faire loi tout ce que la scène anglaise conserve de grossier, d'obscène et d'outré. Mais cela n'empêche pas le déploiement «d'un pathétique noble \& d'une beauté pure» (Suppl., IV, p. 435, b). En plus de la multitude qui ne lit pas, il y a aussi des gens de lettres qui sont les vrai juges devant leurs pairs. Ainsi, «l'on voit deux especes de goût, incompatibles en apparence, se concilier en Angleterre, \& les beautés \& les défauts contraires presque également applaudis» (Suppl., IV, p. 435, b). Cette particularité correspond à «la constitution politique de l'Angleterre» et à «la liberté avec laquelle les Anglois se permettent de parler, de penser \& d'écrire» (Suppl., IV, p. 435, b). L'indépendance et l'originalité dont se piquent tous les individus a, entre autres, pour conséquence, selon Marmontel, que la «poésie philosophique, morale \& satyrique [...] fleurira toujours [en Angleterre], parce qu'elle est conforme au génie de la nation» (Suppl., IV, p. 436, b). Dans le même ordre d'idées, il n'est pas étonnant de remarquer que Marmontel isole le génie de Shakespeare, dont le «mérite réel \& transcendant [...] frappe tout le monde» (Suppl., IV, p. 435, b), et affirme que le «poëme épique de Milton est étranger à l'Angleterre» (Suppl., IV, p. 436, a). Si l'extravagance caractérisait les Espagnols, c'est vraiment la singularité qui caractérise les Anglais.

La singularité est un meilleur dénominateur commun pour tous les Anglais qui peuplent les Nouvelles françaises $d u$ XVIII siècle que leur méchanceté proverbiale. La méchanceté devient véritablement un «exemple unique de fureur et de cruauté» (I, p. 212) dans Trait horrible (1740), une histoire très brève publiée par l'abbé Prévost dans le Pour et le contre. Un officier anglais y assassine de sang-froid sa femme et son voisin. La caste militaire est en général bien représentée parmi les personnages anglais. On retrouve un jeune officier anglais aux prises avec des sauvages dans Abenaki (1765) de Saint-Lambert; quant à William, le narrateur de l'histoire de Caliste (1787) d'Isabelle de Charrière, il avait été destiné «à servir dans l'armée» (II, p. 246) et fut aussi envoyé avec son régiment en Amérique, où il perdit son frère jumeau. Mais, chez SaintLambert et Charrière, les militaires anglais sont en réalité les victimes de leur condition et, s'ils sont singuliers, c'est que leur sort condamne les violences de la guerre. Le bon sauvage abenaki, après avoir sauvé la vie au jeune officier anglais, le renvoie chez son père pour que celui-ci ne souffre pas comme lui de la perte de son fils. Caliste, pour sa part, fait 
tout son possible pour tirer William de la mélancolie, voire de la léthargie dans laquelle l'a précipité son expérience militaire, mais elle n'y parviendra pas. La singularité de cette Anglaise merveilleuse est sans doute de mourir d'amour à cause d'un homme qui est trop inhibé pour se saisir de celle qu'il adore et qui se dérobe au lieu de faire face. Cette histoire d'échec est aux antipodes de celle du Triomphe d'une femme (1736), rapportée par l'abbé Prévost dans le Pour et le contre, où deux amoureux entreprenants sont également éconduits par une belle Anglaise des plus indépendantes. Et que dire du jeune Anglais qui, dans la nouvelle savoyarde (1792) de Florian, abuse de Claudine, alors qu'elle gardait son troupeau sur le Montandverd (II, p. 667-668)? Décidément, les Anglais de nos nouvelles sont imprévisibles, contrairement aux Espagnols très stéréotypés.

Qu'en est-il enfin de la poésie en Allemagne selon Marmontel? Elle aurait dû égaler la poésie lyrique des Anciens, car les Allemands «ont même cet avantage que la musique fait partie de leur éducation commune, \& qu'en Allemagne le peuple même est musicien dès le berceau» (Suppl., IV, p. 436, b). Le poéticien souligne, en effet, des beautés dans les genres de l'ode et de l'églogue. Il cite les Suisses Haller et Gessner et loue l'enthousiasme des odes militaires célébrant le roi de Prusse, mais il déplore pourtant ceci:

Le climat, l'histoire, les mœurs, rien n'étoit poétique en Allemagne; aucune cour n'y a été disposée à élever aux muses des théâtres assez brillans, à présenter assez d'attraits \& d'encouragement au génie, pour exciter dans les esprits cette émulation d'où naissent les grands efforts \& les grands succès (Suppl., IV, p. 437, a).

Quant au poème du Messie de Klopstock et à celui d'Abel de Gessner, Marmontel leur dénie tout comme au Paradis perdu de Milton une spécificité nationale. Ce sont pour lui des poèmes orientaux qui lient entre eux par la croyance tous les peuples d'Europe.

Dans le corpus de nouvelles étudiées, il n'y a que Baculard d'Arnaud qui mette en scène un Allemand dans une nouvelle historique intitulée le Comte de Gleichen (1783). Cette histoire nous ramène au temps des Croisades, sujet qui, selon Marmontel, a fait la gloire de la poésie italienne. Mais Baculard d'Arnaud n'explore pas le merveilleux, il explore la sensibilité des êtres. Il a travaillé son sujet à partir de plusieurs sources et il reformule l'histoire du preux chevalier qui échappe à sa captivité en Orient grâce au grand amour que lui voue Zélide, la fille du roi dont il était le prisonnier. Enfin libre et en route pour sa patrie, Gleichen est plongé dans une mélancolie mortelle, car il a omis de dire à sa princesse qu'une femme et des enfants l'attendent en Allemagne. Si son caractère ressemble à celui des gens de sa nation naturellement 
portés «à des méditations profondes» (Suppl., IV, p. 437, a), ce trait est rendu plus sensible par contraste avec son compagnon de captivité grec, qui incarne, pour sa part, la fourberie proverbiale des Grecs. C'est lui qui a convaincu Gleichen de se taire pour que la petite troupe de prisonniers puisse échapper à la captivité. Mais le chevalier allemand s'en plaint amèrement, car son âme est tourmentée et il craint également les tourments que vivront les deux femmes de son cœur:

Voilà donc où vous avez amené un chevalier! à trahir la probité, l'honneur, la religion, car je l'offense, cette religion de vérité, en me servant de l'artifice, du mensonge, du mensonge si bas, si vil, si dégradant pour un rejeton des Swartzbourgs! (II, p. 519)

Dans cette nouvelle, l'abondance des larmes, des scrupules délicats et des beaux sentiments donne des Allemands une image bien éloignée du préjugé selon lequel ce sont de grossiers ivrognes. Comme le soulignait Marmontel dans son histoire naturelle de la poésie, tant pour les Anglais que pour les Allemands, la rudesse de la langue n'empêche pas l'expression des vérités naïves du cœur, dont la beauté poétique a son prix vers la fin du XVIII ${ }^{\mathrm{e}}$ siècle, et revalorise les identités nationales du Nord, que l'on pense aux Anglais, aux Allemands, mais aussi aux Suisses et aux Suédois.

Pour conclure, on peut constater que les identités étrangères dans les Nouvelles françaises $d u$ XVIII siècle reflètent parfois les lieux communs concernant les caractères nationaux, tels qu'on se plaît à les répéter à la manière de proverbes. Mais cette première surface est souvent enrichie de traits qui présupposent une connaissance des œuvres marquantes issues des nations mises en jeu et de la place qu'elles ont ainsi conquise dans l'édifice de la culture européenne. L'article «Poésie» que Marmontel imagine au milieu des années soixante-dix sert bien sûr à situer favorablement la voix de la nation française dans ce concert, à rappeler l'intérêt universel de ses grands auteurs et l'émulation bénéfique de la scène parisienne. Mais il redéfinit aussi la perception qu'on doit avoir des autres identités nationales d'une façon qui trouve des échos dans la caractérisation des personnages étrangers des nouvelles étudiées. $S^{\prime}$ il m'est permis de revoir maintenant les caractères nationaux à la lumière de cette recherche, je dirai avec les voix qui se font entendre à partir des années 1760 et 1770: mystérieux comme un Italien, extravagant comme un Espagnol, singulier comme un Anglais, profond comme un Allemand et supérieur comme un Français. 